\title{
Infective endocarditis in South Africa
}

\author{
Alfonso Jan Pecoraro, Anton Frans Doubell \\ Division of Cardiology, Department of Medicine, Stellenbosch University and Tygerberg Hospital, Cape Town, South Africa \\ Contributions: (I) Conception and design: All authors; (II) Administrative support: All authors; (III) Provision of study materials or patients: None; \\ (IV) Collection and assembly of data: None; (V) Data analysis and interpretation: None; (VI) Manuscript writing: All authors; (VII) Final approval of \\ manuscript: All authors. \\ Correspondence to: Alfonso Jan Pecoraro. Division of Cardiology, Department of Medicine, Stellenbosch University and Tygerberg Hospital, Cape \\ Town, South Africa. Email: pecoraro@sun.ac.za.
}

\begin{abstract}
Infective endocarditis (IE) is defined by infection of either a native or prosthetic heart valve, endocardial surface or any cardiac prosthetic device. It is a disease of both the developed and developing world, although IE has evolved in the developed world to a disease markedly different to the disease encountered in the developing world. IE in developed nations is mainly a disease of older patients with degenerative heart valve disease or cardiac prosthetic material, with virulent Staphylococci the most common causative organism. Data regarding the epidemiology of IE in South Africa, a developing country, is limited. The available data suggest it is still a disease of younger patients with rheumatic heart disease (RHD), associated with penicillin-sensitive Streptococcal infection. Although novel diagnostic techniques and improved therapeutic options has emerged, the 1-year mortality rate has remained high in both the developed and developing world at around $30 \%$.
\end{abstract}

Keywords: Infective endocarditis (IE); rheumatic heart disease (RHD); valvular heart disease

Submitted May 14, 2019. Accepted for publication Jun 12, 2019.

doi: $10.21037 / \mathrm{cdt} .2019 .06 .03$

View this article at: http://dx.doi.org/10.21037/cdt.2019.06.03

\section{Patient profile}

IE is a rare disease affecting 3-10/100,000 patients per year with recent data from the United States of America (USA) and United Kingdom suggesting a rise in the incidence $(1,2)$. Data regarding the incidence in South Africa is lacking. It is well documented that the presence of rheumatic valvular heart disease significantly increases patient's risk of acquiring infective endocarditis (IE) (3). The fact that South Africa still has a high prevalence of rheumatic heart disease compared to the developed world, puts a significant part of the population at risk for developing IE. The developed world, predominantly the USA and Europe, are experiencing IE as an "old disease in a new guise". In the pre-antibiotic and early antibiotic era, IE was a disease of young patients with rheumatic heart disease (RHD) or congenital heart disease (1). This has evolved to a disease of older patients in developed nations due to an increase in the life expectancy of the population, the decrease in RHD, increase in availability of antibiotics coupled with increased admission to health care facilities and the advent of modern cardiology where device therapy plays a significant role $(1,4)$.

IE in South Africa is still strongly associated with RHD and thus the patient profile is that of young to middle age adults with RHD. A prospective observational trial of patients with IE conducted at Tygerberg Hospital, Cape Town, South Africa reported a mean age of 37.7 years- $72 \%$ of patients had underlying RHD (5). A more recent study conducted in Cape Town, South Africa revealed similar results with up to $80 \%$ of patients with NVE having evidence of underlying RHD (6). The Echoin-Africa (EIA) study screened over 6,000 secondary school pupils in the Tygerberg Hospital referral network (Cape Town, South Africa) and found a prevalence of 20/1,000 of pupils screened to have borderline or definite RHD, as per the World Health Federation criteria (7) for the detection of RHD (unpublished data). This leaves a large 
part of the population at significant risk for IE. The rise of interventional cardiology in South Africa with increased access to not only cardiac care but health care in general bring to the fore a possible group of patients with IE not generally associated with developing nations (8). It is safe to predict that the characteristics of IE as experienced by developed nations will increase in the South African setting as life expectancy and access to medical care increases.

\section{Microbiology}

The change in patient profile and risk factors for IE has coincided with a change in microbiology of IE. Staphylococci, usually associated with health-care contact and invasive procedures has overtaken Streptococci as the most common causative organism in developed nations $(1,4,9)$. The significant incidence of culture negative endocarditis observed in South African cohorts raises questions regarding the true microbiology of IE in South Africa $(5,6)$. It must be noted that these reports predate modern algorithms of microbe detection as well as modern imaging that enable earlier detection and more accurate diagnosis.

Streptococcal IE caused by the oral viridans group remains most common in low-income countries and is the most common cause in reported literature in South Africa (2-4). The fact that rheumatic valve disease is common in the developing world coupled with poor oral hygiene creates the perfect conditions for the development of IE. These commensals of the oral cavity gain access to the blood stream via the mucosa resulting in a bacteraemia. Patients with underlying RHD with regurgitant lesions provide the perfect nidus for developing IE in patients with a bacteraemia. The viridans group includes a host of organisms including Streptococcus mutans, Streptococcus salivarius, Streptococcus anginosus, Streptococcus mitis and Streptococcus sanguinis (the term "viridans" comes from the Latin word for the colour green-the colour of this group on blood agar) $(1,4)$.

Staphylococcus aureus is the most frequent organism isolated in the developed world cohort. Staphylococcal IE has the ability to effect both normal and abnormal native valves, as well as prosthetic valves/material (4). Staphylococcus aureus associated IE is commonly encountered in intravenous drug abusers and patients with long term indwelling catheters e.g., patients on haemodialysis; but are now often encountered in patients that are not from these groups. In South Africa, Staphylococcal IE is often seen in intravenous drug abusers.
A recent study of patients with IE related to intravenous Nyaope abuse, a drug unique to some parts of South Africa, reported Staphylococcus aureus positive cultures in $61 \%$ of patients (10).

Coagulase-negative Staphylococci (e.g., Staphylococcus epidermidis, Staphylococcus lugdunensis and Staphylococcus capitis) are ubiquitous skin commensals. They colonise indwelling lines and devices and are the most common cause of early prosthetic valve endocarditis. Coagulase-negative staphylococci also frequently cause hospital-acquired native valve endocarditis. All forms of staphylococcal IE have a propensity for developing antibiotic resistance (11).

Other causative organisms tend to occur in specific patient populations and should be considered in certain clinical settings. An example of such a group is Enterococci (up to $10 \%$ of IE), of which most isolates are Enterococcus faecalis. These organisms typically cause IE in chronically ill patients and the elderly. In patients where group D Streptococci (Streptococcus gallolyticus, Streptococcus bovis) are isolated, an underlying colonic tumour should be suspected (4).

The remaining microbes that can cause IE are a mixture of fastidious bacteria, zoonotic bacteria and fungi, often responsible for so called blood culture negative IE (BCNIE). The HACEK bacteria (Haemophilus, Aggregatibacter, Cardiobacterium, Eikenella and Kingella), which cause about $3 \%$ of cases, are slow-growing organisms that colonise the oropharynx. Zoonotic endocarditis is caused by Coxiella burnetii and Brucella (from livestock), Bartonella henselae (from cats) and Chlamydia psittaci (from parrots, pigeons). It is important to note that the prevalence of these groups were much lower in South African cohorts compared to developed countries (2-4). Fungal endocarditis due to Candida or Aspergillus, is rare but often fatal, arising in patients who are immunosuppressed or after cardiac surgery $(4,11,12)$. It is interesting to note that fungal associated IE is not commonly encountered in patients with human immunodeficiency virus (HIV), even in the advanced stages of the disease $(13,14)$.

\section{Clinical features}

The clinical features of IE have changed with a decrease in the "classical signs" of IE. The majority of patients present with fever $(90 \%)$, frequently associated with chills, poor appetite and weight loss. Most patients (>85\%) will have some form of heart murmur on auscultation, with about 
$25 \%$ of patients presenting with embolic phenomena (11). It is therefore common teaching that IE should be strongly suspected in any patient with fever and embolic phenomena. It is reported that the other classical vascular and immunological phenomena are mostly seen in the developing world, mostly due to patients presenting late and the difference in causative organisms (1). However, splinter haemorrhages, clubbing and glomerulonephritis are still commonly seen in South Africa (5). The elderly and immunocompromised patients may not present with the typical feature of fever, as fever is less common than in younger patients (11). Right sided IE, often seen in intravenous drug abusers, immunosuppressed patients, patients with indwelling lines or pacemaker leads, has the additional complication of septic emboli to the lungs. These patients may in addition to the typical features of left sided IE (barring systemic emboli) present with recurring respiratory infections and pulmonary hypertension. Right sided IE is typically caused by Staphylococci. The chest $\mathrm{X}$-ray of patients with septic emboli to the lungs usually shows multiple, bilateral areas of opacification with air-fluid levels due to tissue breakdown.

It is our experience that acute heart failure with or without cardiogenic shock is another relatively common mode of presentation. Acute heart failure is mostly due to acute or acute on chronic left sided regurgitant lesions. Patients with acute aortic/mitral regurgitation pose a particular clinical challenge as the hallmark of severe regurgitant lesions namely apex beat displacement/ ventricular enlargement is absent and most patients do not have the typical peripheral signs of severe aortic/mitral regurgitation (15). Acute mitral regurgitation (MR) can be extremely challenging as the only clinical sign can be a faint, short systolic murmur due to rapid equalisation of pressure in the left ventricle and atrium. Acute aortic regurgitation (AR) is usually marked by a low pulse pressure with the typical short diastolic murmur due to rapid equalisation of aortic and left ventricular pressures during early diastole. This is often referred to as the "inverse cadence", due to the very short duration of the diastolic murmur it might be mistaken for a systolic murmur. The realisation that a murmur, initially thought to be systolic is actually diastolic after timing it with the carotid pulse, is typical of the inverse cadence of acute severe AR. It is paramount that clinicians have a high index of suspicion when examining patients with acute heart failure and an undisplaced apex beat as correctly diagnosing acute regurgitant lesions might be as challenging with echocardiography as it is with examination.

\section{Identification of the causal organism}

The cornerstone of the diagnosis of IE is based on the Modified Duke/ESC 2015 Modified Criteria (11). The hallmark of the diagnosis of IE is:

- Blood cultures identifying organisms that explain the clinical and imaging findings;

* Features of IE on either transthoracic or transoesophageal echocardiography (TEE), although cardiac computer tomography (CT) with or without 18 -fluorodeoxyglucose positron emission tomography $\left({ }^{18}\right.$ FDG-PET) and leukocyte scintigraphy [radiolabelled-leukocyte single-photon emission computed tomography (SPECT)] may be useful in specific cases.

Blood cultures that are negative in patients with imaging and/or clinical evidence of IE can pose both a diagnostic and therapeutic challenge. Unfortunately, BCNIE is relatively common in most series, with a reported rate of $10 \%$ to $30 \%(4,5,11)$. A study conducted at Tygerberg Hospital, Cape Town, South Africa found a BCNIE rate of more than $50 \%$ in a cohort of patients with definite IE. The majority of these patients were treated with antibiotics prior to blood cultures taken and this was proposed to be the major contributor to the very high incidence of BCNIE (5). Recent reports from South Africa have revealed BCNIE in $30 \%$ of patients $(6,10)$. The etiology of BCNIE varies according to region, reflecting differences in local pathogens, initiation of antibiotics prior to taking blood cultures, inadequate blood culture sampling and incorrect interpretation of imaging findings. This does raise the question whether the very high incidence of BCNIE in South Africa is due to local unidentified pathogens or rather related to the combination of early antibiotic use, inadequate blood culture sampling and the lack of use of polymerase chain reaction (PCR) panels of heart valve tissue and blood. To blame early antibiotic use in a resource limited section as the only cause for the high rate of BCNIE does not seem logical.

The contemporary evaluation of patients with BCNIE aims at identifying pathogens that are either nonculturable or difficult to culture (slow growing or requiring special growth media). The ESC guidelines on IE propose a specific diagnostic algorithm which has led to a significant reduction in the number of BCNIE patients (11). This algorithm suggests further testing in the form of serology and to proceed with PCR of either blood or heart valve tissue should this be negative for patients with suspected BCNIE. 

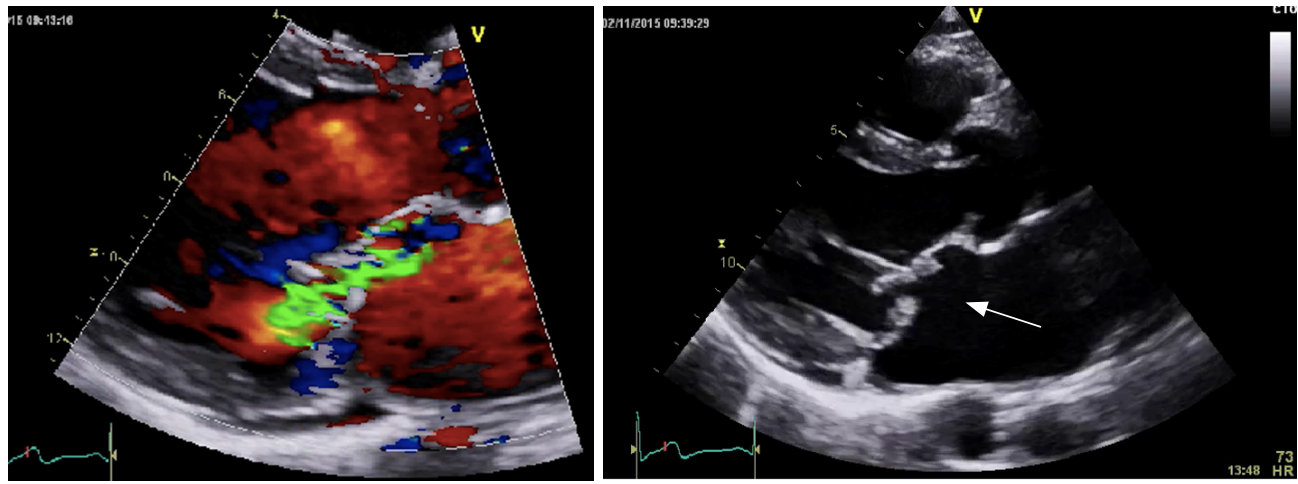

Figure 1 Vegetations (arrow) visible on the atrial surface of the mitral valve within the jet of mitral regurgitation.

In a large prospective study of 759 patients with BCNIE, a systematic diagnostic protocol identified a causative organism in $62 \%$ of patients. Of the patients where a causative organism was identified, blood serology had the highest yield with $75 \%$ of the identified organisms either Coxiella or Bartonella species. Polymerase chain reaction (PCR) assays of the heart valves had the second highest yield with $66 \%$ of patients with negative serology testing positive by $16 \mathrm{~S}$ rDNA assays. Polymerase chain reaction assays of the blood (16S rDNA assays) were positive for $13.6 \%$ of patients with negative serology. Auto-immunohistochemistry had a much lower diagnostic yield than any of these modalities $(16,17)$.

The most common organism detected in developed countries in the BCNIE group is Coxiella Burnetti, although not a single case was detected in a previous study in South Africa $(5,17)$. Of interest is the finding of predominance of Bartonella species in a similar study in Algeria, a developing country similar to South Africa (17).

Imaging plays a key role in the diagnosis of IE. Currently 4 imaging modalities are validated for the diagnosis of IE namely (1):

* Transthoracic echocardiography (TTE);

* TEE;

* Cardiac computer tomography (CT);

* ${ }^{18}$ FDG-PET/CT or leucocyte labelled SPECT.

\section{Echocardiography in IE}

TTE is the recommended initial modality of choice for both native valve IE (NVE) and prosthetic valve IE (PVE). The role of both TTE and TEE is to detect features of IE, assess the underlying valvular abnormality (if present) and to assess the haemodynamic effect.

The finding of independently mobile masses or vegetations on heart valves is the hallmark finding on echocardiography (TTE/TEE). These are usually found down-stream to regurgitant jets on the atrial surface of the mitral/tricuspid valve (Figure 1) and ventricular surface of the aortic/pulmonic valve. Varying degrees of valve destruction is another typical finding in patients with IE depending on the virulence of the organism. The notable exception is fungal endocarditis where large vegetations with high embolization risk are found with minimal tissue destruction. Mycobacterium tuberculosis, a rare cause of IE, is sporadically encountered in the Western Cape area of South Africa, an area with a very high incidence of pulmonary tuberculosis $(18,19)$. The imaging findings are unique with no or very small vegetations seen on the edges of leaflets with significant tissue destruction. Tissue destruction is usually located to the body of the mitral valve leaflet (rather than the edges) and perforation is common (Figure 2). The presence of other features of myocardial tuberculosis i.e., submitral aneurysm or aortic aneurysms further raises the suspicion of tuberculous endocarditis.

Peri-annular extension of infection, particularly abscess or fistula formation is more common in the aortic position (in NVE) or in patients with PVE and usually associated with Staphylococcal infection.

Echocardiography (TTE in particular) is also very useful for evaluating the underlying valvular structure. It is our experience that patients with evidence of previous rheumatic valvular involvement are more likely to have streptococcal associated IE in contrast to patients with normal underlying heart valves that mostly have staphylococcal associated IE.

In patients with suspected NVE, TTE has a higher sensitivity (50\% to $90 \%$ ) and a specificity $(90 \%)$ than in patients with PVE (sensitivity of $40 \%$ to $70 \%$ ), yet even in 


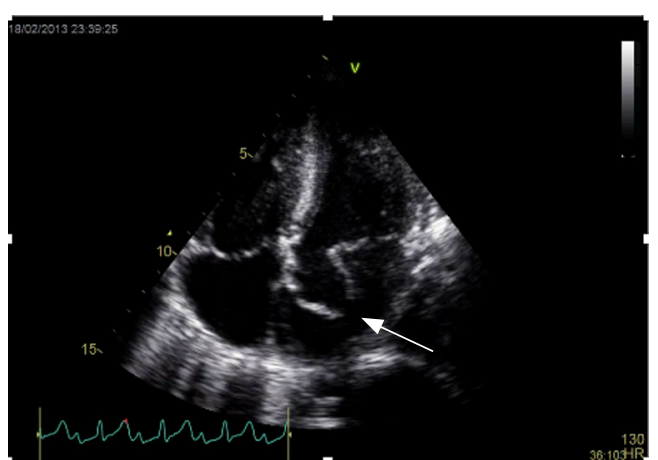

Figure 2 Apical four chamber view of a patient with confirmed tuberculous endocarditis with aneurismal perforation of anterior mitral valve leaflet (arrow).

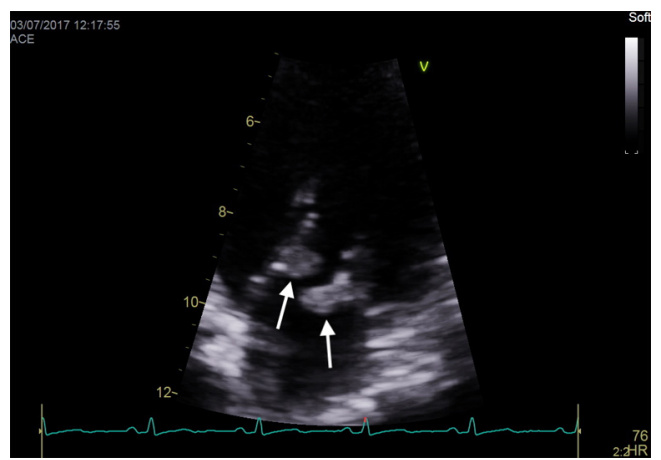

Figure 3 "Kissing vegetations" (arrows) on adjacent leaflets of the mitral valve typical of NBTE. NBTE, non-bacterial thrombotic endocarditis.

PVE it provides value in assessment of ventricular size and function, hemodynamic severity of valve lesions and in the diagnosis of anterior prosthetic aortic/mitral valve abscesses. Due to the shielding effect of prosthetic valves, abscess formation anterior to aortic/mitral valve is better visualized via TTE than TEE $(1,11)$. TEE is indicated in almost all patients with suspected IE. For suspected NVE, TEE has a high sensitivity $(>90 \%)$ and specificity $(>90 \%)$ for detection of vegetations. TEE has been demonstrated to be superior to TTE for detection of complications such as perforations, abscesses and fistulae. In clinical practice TTE and TEE are used as complementary modalities to accurately diagnose and assess patients with suspected IE.

All valvular lesions should be quantified according to current guidelines, but the echocardiographer should be on the lookout for the haemodynamic features of acute severe regurgitant lesions as it will necessitate emergency/urgent surgery $(11,20)$. As with the clinical evaluation, the typical feature of ventricular dilatation is not present.

An important clinical and echocardiographic mimicker of IE and one of the causes of BCNIE is non-bacterial thrombotic endocarditis (NBTE). Patients with NBTE typically present with embolic events in the absence of stigmata of IE and during echocardiography are found to have a vegetation/s. The presence of so-called "kissing vegetations" on adjacent leaflets of either the mitral/aortic valve without significant valvular destruction should raise suspicion (Figure 3). NBTE can be associated with a host of diseases of which primary antiphospholipid syndrome, systemic lupus erythematosus (so called Libman-Sachs endocarditis) or malignancy are the most common.

\section{Adjunctive imaging modalities}

Although TEE has a high sensitivity (86\%) in PVE (depending on the operator), newer imaging modalities are emerging to help confirm or exclude the diagnosis in cases in where a combination of TTE/TEE is nondiagnostic. These newer imaging modalities for IE are most useful in the diagnosis of PVE and both cardiac CT and ${ }^{18}$ FDG-PET/CT or leucocyte labelled SPECT has been validated in this setting (11). Cardiac CT is a key adjunctive modality for use when the anatomy is not clearly delineated by echocardiography. Cardiac CT is equivalent to echocardiography for the detection of paravalvular anatomy and possible complications e.g., abscess formation. Combining CT imaging with metabolic imaging by ${ }^{18} \mathrm{FDG}-$ PET or leucocyte labelled SPECT has the added benefit of detecting both anatomic and metabolic abnormalities in patients with suspected IE $(1,11,21)$. During the initial three months after prosthetic valve replacement, ${ }^{18} \mathrm{FDG}-\mathrm{PET} / \mathrm{CT}$ or leucocyte labelled SPECT should not be utilised as falsepositives due to perivalvular inflammation related to surgery occur (11). Current data is insufficient to define a diagnostic role for cardiac MRI (21).

Patients evaluated for IE should follow the diagnostic algorithm as proposed by recent guidelines and evaluated by the endocarditis team (11). Once all indicated investigations are interpreted in the context of the clinical presentation, patients with definite IE should be treated as such and those with rejected IE the alternative diagnosis treated. A separate group, so called probable IE, exist where the current diagnostic criteria are not met but the endocarditis team is of the opinion enough features exist to warrant treatment for IE (11). 


\section{Management of IE}

The management of patients with IE aims to eradicate infection and restore cardiac structure. Eradicating infection, whether medically or surgically, should abort the disease process and thus prevent further local, hemodynamic, immunologic or embolic complications. Structural restoration is primarily surgical, aimed at repairing damaged tissue that has a low likelihood of spontaneous healing and would otherwise have short- or long-term hemodynamic effect $(11,22)$.

\section{Antimicrobial therapy}

In terms of eradication of microbes, the host defenses are inadequate. This explains why bactericidal regimens are more effective than bacteriostatic therapy, both in animal experiments and in humans. Aminoglycosides have a synergistic effect in combination with cell-wall inhibitors (beta-lactams and glycopeptide) for bactericidal activity and are useful for shortening the duration of therapy (e.g., oral Streptococci) and eradicating problematic organisms (e.g., Enterococci) (11). Pathogen-specific recommendations for antibiotics are complex and are well summarized in a recent guideline (11). Optimal therapy of IE requires bactericidal antibiotics for a prolonged period, with initiation of empiric antibiotics as soon as adequate blood culture samples have been acquired. Three sets of blood cultures from different sites should be acquired at 30-min intervals before initiation of antibiotics (11).

The initial choice of empirical treatment depends on several considerations. The decision regarding empirical therapy depends on the most likely causative organism or group of organisms. In addition, knowledge of the local epidemiology, especially for antibiotic resistance and specific genuine culture-negative pathogens is very important. Currently little is known regarding resistance profiles and pathogens not routinely detected by blood culture in the South African context.

Other factors that influence the choice of empirical therapy are:

* The valve affected-NVE versus PVE (any prosthetic material e.g., pacemaker lead infection is classified as PVE);

* The likely mode of infection.

In general, patients with NVE with no recent admission to hospital or health care facility and no history of IV drug abuse are treated with a combination of ampicillin/penicillin and gentamicin with cloxacillin $(11,22)$. The aim is to cover Streptococci, Staphylococci and Enterococci. Due to the lower rates of community acquired Staphylococcus aureus associated IE in South Africa, some institutions will treat this group without the addition of cloxacillin (5). This underlines the importance of population specific data on causative microbes in the setting of IE as it influences empirical antibiotic regimes.

A very important consideration in PVE is the time since surgery. Early PVE (within 60 days of surgery) is typically caused by hospital acquired pathogens or coagulase negative Staphylococci (from the skin) and thus the empirical regimen should in addition to the normal pathogens cover methicillin resistant Staphylococci and non-HACEK gram negative pathogens. Community acquired PVE more than 60 days post-surgery typically has the same pathogen profile as NVE and as thus the empirical regimen is similar to NVE. In patients with PVE, rifampicin should be introduced at a time when the bacteraemia has cleared, usually 3 to 5 days after initiation of antibiotic therapy. The rationale supporting the initiation of rifampicin is based on the likely antagonistic effect of the antibiotic combinations with rifampicin against planktonic/replicating bacteria and the synergy seen against dormant bacteria within the biofilms on prosthetic valves. The introduction after clearing of the bacteraemia is to prevent rifampicin resistant strains developing, an extremely important consideration in areas with a high prevalence of tuberculosis (11).

Once the pathogen is identified (usually within $48 \mathrm{~h}$ ), the antibiotic treatment must be adapted to its antimicrobial susceptibility pattern. A recent important change to the treatment of Staphylococcal IE is the fact that aminoglycoside co-therapy for its synergistic effect is not recommended. No clinical benefit has been demonstrated with this strategy and the use of aminoglycosides increases the incidence of renal failure due to renal toxicity (11). Typical regimens for antimicrobial treatment consist of 4 to 6 weeks of intravenous (IV) antibiotics, but a recent publication suggest the feasibility of initial 10-14 days of IV therapy followed by outpatient oral therapy $(11,23)$. Stable patients with culture positive IE were treated for a minimum of 10 days with IV antibiotics and then changed to oral antibiotics with no adverse effect on all-cause mortality, relapse, urgent surgery or embolic events (23). This has not been formally tested in a South African health care setting, but the high rates of BCNIE will significantly reduce the number of patients eligible for this strategy. These results of outpatient therapy for IE should be 
interpreted with caution as this is a single Danish study and might not be reproducible in developing nations.

\section{Surgical therapy}

Approximately half of patients presenting with IE in the developed world, require surgery $(22,24)$. This is similar to the rates reported for patients in South Africa $(5,6)$, although our current experience is that the majority of patients will require surgery.

The indications and timing of surgery in patients with IE revolve around three key factors namely heart failure, embolic risk and infection control. The timing of surgery can be divided into emergency ( $<24 \mathrm{~h})$, urgent ( $<7$ days) end elective (>7 days) (11).

The most common indication for surgery in patients with left sided IE is destruction of heart valves with subsequent heart failure. Patients with cardiogenic shock or refractory pulmonary oedema, mostly due to acute or acute on chronic aortic/mitral regurgitation, should be scheduled for emergency surgery. Patients with ongoing symptoms of heart failure with echocardiographic evidence of poor haemodynamic tolerance should receive early surgery. In patients with well-tolerated (New York Heart Association class I or II) heart failure with severe valvular regurgitation (not acute severe) and no other reasons for surgery, medical management with antibiotics under strict clinical and echocardiographic observation is the current standard of care, although early surgery may be an option in selected patients at low risk for surgery. Elective surgery should be considered depending on the tolerance of the valve lesion and according to the recommendations of the ESC guidelines on the management of valvular heart disease $(11,20)$.

The prevention of embolic phenomenon, especially cerebrovascular accidents (CVA) is another important indication for early surgery in IE of left sided valves. Overall, embolic risk is very high in IE, with embolic events occurring in $20 \%$ to $50 \%$ of patients. However, the risk of new events (occurring after initiation of antibiotic therapy) is significantly lower at $6 \%$ to $21 \%$. It has been demonstrated that the incidence of stroke in patients receiving appropriate antimicrobial therapy was 4.8/1,000 patient-days in the first week of therapy, falling to $1.7 / 1,000$ patient-days in the second week, and further thereafter (25). Current best practice is to offer early surgery if the vegetation is larger than $15 \mathrm{~mm}$ in diameter. For patients with vegetations ranging from $10-15 \mathrm{~mm}$, an individualized approach is followed (24). In this category of patients, the presence of thromboembolic events e.g., transient ischemic events and/or severe mobility of the vegetation will favour early surgery. Should the vegetation size increase in spite of antimicrobial therapy, patients should be operated early as the risk of systemic embolization is high (11).

An area of conflicting data is that of timing of surgery for patients that present with an ischemic stroke. Multiple studies have confirmed the risk of neurological deterioration due to haemorrhagic transformation should valvular surgery be performed during the initial days after the stroke. One such study calculated the risk of neurological deterioration as $35 \%$ on day one, $15 \%$ on days two and three and $20 \%$ to $50 \%$ from day 4 to day 14 . After 14 days the risk was lower than $10 \%$ and after 4 weeks it was $0.4 \%(24,26)$. This would suggest that waiting 4 weeks post ischemic stroke should significantly reduce the risk of decline in neurological function due to haemorrhagic transformation. It is however offset by this risk of further thromboembolic events during the delay to surgery. In patients with embolic complications, the rate of recurrent embolism is markedly elevated with studies reporting a rate of up to $50 \%$. The majority of these occur during the first 18 days after the initial event and $80 \%$ occurred within 32 days. This provides some insight into the time-dependent risk of recurrent embolization with delayed surgery (26). The current best practice would be a multidisciplinary team approach (endocarditis team) where the risk of neurological exacerbation due to surgery is balanced by the risk of recurrent embolic events $(11,24,26)$.

Less commonly, the inability to control the infection (persistent fevers) or the development of perivalvular complications e.g., abscess or fistula formation will necessitate surgery. The frequency of fistula formation in IE has been reported to be $1.6 \%$, mostly associated with Staphylococcal IE (11). Patients with IE due to microorganisms at low likelihood of being controlled with antimicrobial therapy, such as fungal IE, multi-resistant organisms or rare cases of IE due to Gram negative bacteria, should be considered for early surgery. Patients with PVE due to Staphylococci should also be considered for early surgery (11).

In general, patients with isolated right sided endocarditis are less likely to be referred for surgery. The initial surgical mortality of these patients is lower than for left sided IE, but the reinfection rate is high mostly due to the fact that most patients with isolated right sided endocarditis are IV drug abusers. Recent data has challenged this. Most patients readmitted after surgery for right sided IE are due to repeat abuse of drugs and not reinfection, although 
reinfection is still higher than with left sided IE $(11,27)$. The indication for surgery in isolated right heart IE follows the same principles as left sided IE. Patients with severe right heart failure due to severe tricuspid regurgitation that does not respond to diuretic therapy, large vegetations more than $20 \mathrm{~mm}$ with recurrent septic pulmonary emboli and patients with persistent bacteraemia ( $>7$ days) are the usual indications for surgery (11).

Surgery for IE revolves around eradicating infection and debriding all necrotic tissue, with subsequent reconstruction of the cardiac chambers and valves. The reconstructive material should ideally provide durability and a low reinfection rate. In general, repair of valves or cardiac structures is more challenging in patients with IE. The technical difficulty increases with peri-annular extension which is proportional to the virulence of the causative organism. The presence of underlying structural defects e.g., RHD or bicuspid aortic valve disease further decreases the chances of a successful, lasting repair. Every effort should be made to avoid implanting a prosthesis. In some cases, implanting a prosthesis becomes inevitable because of extensive tissue destruction and bioprosthesis are preferred over mechanical valves by some authors. This is largely based on observational studies and each case should be individualized $(11,16)$. Homografts and stentless valves may provide valvular support when the perivalvular tissue is extensively destroyed (22). The implantation of bioprosthesis is mostly a trend in the developed world where patients are older at presentation and have access to percutaneous valve-in-valve procedures should there bioprosthesis degenerate. In the developing world and South Africa in particular, the current practice is to use mechanical prostheses if conventional repair is not possible (5). Conventional repair for patients with IE are in the minority as most patients have extensive valvular destruction due to IE with underlying rheumatic valve involvement. In a recent 5 -year retrospective audit of mitral valve repair at Tygerberg Hospital (Cape Town, South Africa), only a small number of patients with IE had successful mitral valve repair (28). A new emerging alternative for patients not suitable to conventional repair is reconstruction of the anterior mitral valve leaflet (AMVL) with autologous venous tissue $(29,30)$. The fact that no anticoagulation is required provides this approach with an attractive advantage for younger patients, although longevity and freedom from reoperation is still to be proven. An additional potential advantage might be the rate of reinfection in patients with IE. Reinfection rates are very low with conventional repairs and it is reasonable to predict that reinfection rates should be lower with this approach compared to mechanical valve replacement (31). A recent review of mitral valve repairs at Tygerberg Hospital (Cape Town, South Africa) reported no patients with relapse/reinfection in patients with IE that underwent mitral valve repair within 6 months of surgery (28).

\section{Recurrence and prevention}

Patients who survive the initial therapy including surgery if indicated, are at risk for recurrence. The actual risk of recurrence among survivors of IE varies between $2 \%$ and $6 \%$. Recurrence of IE can be divided into relapse or reinfection. The term 'relapse' refers to a repeat episode of IE caused by the same microorganism, while 'reinfection' describes an infection caused by a different microorganism. When the same species is isolated during a subsequent episode of IE, there is often uncertainty as to whether the repeat infection is due to relapse of the initial infection or a new infection (reinfection). Proven infection with the same species within 6 months represents relapse, whereas later events suggest reinfection. The common causes of relapses are due to insufficient duration of original treatment, suboptimal choice of initial antibiotics or a persistent focus of infection (4).

Patients with previous IE are at risk of reinfection and prophylactic measures should be very strict. Reinfection is more frequent in intravenous drug abusers (especially in the first year after the initial episode), PVE in patients undergoing chronic dialysis and in those with multiple risk factors for IE. Although the recurrence rate is much lower in patients with valve repair surgery, no clear difference in terms of recurrence is reported when the valve is replaced with either biological or mechanical prosthesis. Good oral health maintenance, preventative dentistry and advice about skin hygiene, including tattoos and skin piercing, are mandatory (11).

Patients with previous endocarditis, prosthetic valves or material and those with uncorrected congenital heart disease (atrial septal defects excluded) are at higher risk for IE after dental procedures and should receive prophylaxis as per current guideline (11). The role of antibiotic prophylaxis before dental procedures that requiring manipulation of the gingival or peri-apical region of the teeth, has been disputed by several authors. There are no randomised controlled trials to support a specific strategy and it is unlikely such a trial will be performed. The pathogenesis of Streptococcus viridans IE, especially in the RHD population, is well documented to be due to entry 
into the blood stream via the gingivae. Although low grade bacteraemia is common during normal dental activities e.g., tooth brushing, the levels of bacteraemia is much higher during invasive dental procedures $(1,3)$. Recent observational studies from developed nations (Europe, USA and UK) after the restriction of antibiotic prophylaxis has yielded conflicting data. Reports from France and the USA has demonstrated no increase in the incidence of IE after the change in guidelines, while the UK has seen a significant increase in cases of IE after restriction of antibiotic prophylaxes for dental procedures $(1,4,9,32)$. Unfortunately, it is not known whether the increase in IE in the UK was due to an increase in cases of Streptococcus viridans associated IE. The South African Heart association has cautioned against introducing these guidelines locally, as the data used to support the restriction of antibiotic prophylaxis was derived from populations with a low incidence of RHD (3). In a recent position statement, the South African Heart association still recommends antibiotic prophylaxis for patients with RHD and specifically for those with left sided regurgitant lesions in addition to the current ESC guideline recommendation $(3,11)$.

The prevention of IE in South Africa focuses on four main areas namely:

* Improvement in general socio-economic state of the population;

* Early detection and treatment of subclinical RHD;

* Improving oral health;

* Limiting the use of prosthetic material in patients undergoing valve surgery.

The majority of young patients undergoing cardiac surgery for severe valve disease will receive a mechanical prosthesis due to the high incidence of RHD associated valve disease in South Africa. This leaves this group of patients with a lifetime risk of developing PVE in addition to the burden of anticoagulation and the expected attrition rate of a prosthetic valve. A strategy of reducing prosthetic valve replacement in favour of either repair or reconstruction with living tissue should significantly reduce relapse/reinfection rates as well as PVE in patients with/without previous IE.

\section{Conclusions}

The clinical features and microbiology of IE on the African continent, and in particular South Africa, has distinct differences from the patient population encountered in developed nations. This presents a unique opportunity to improve patient outcomes with innovative prevention and intervention strategies. The collection of contemporary data to guide future strategies is of the utmost importance.

\section{Acknowledgments}

Funding: None.

\section{Footnote}

Provenance and Peer Review: This article was commissioned by the Guest Editor (Ntobeko A. B. Ntusi) for the series "Cardiovascular Diseases in Low- and Middle-Income Countries" published in Cardiovascular Diagnosis and Therapy. The article was sent for external peer review organized by the Guest Editor and the editorial office.

Conflicts of Interest: Both authors have completed the ICMJE uniform disclosure form (available at http://dx.doi. org/10.21037/cdt.2019.06.03). The series "Cardio-vascular Diseases in Low- and Middle-Income Countries" was commissioned by the editorial office without any funding or sponsorship. The authors have no conflicts of interest to declare.

Ethical Statement: The authors are accountable for all aspects of the work in ensuring that questions related to the accuracy or integrity of any part of the work are appropriately investigated and resolved.

Open Access Statement: This is an Open Access article distributed in accordance with the Creative Commons Attribution-NonCommercial-NoDerivs 4.0 International License (CC BY-NC-ND 4.0), which permits the noncommercial replication and distribution of the article with the strict proviso that no changes or edits are made and the original work is properly cited (including links to both the formal publication through the relevant DOI and the license). See: https://creativecommons.org/licenses/by-nc-nd/4.0/.

\section{References}

1. Cahill TJ, Baddour LM, Habib G, et al. Challenges in Infective Endocarditis. J Am Coll Cardiol 2017;69:325-44.

2. Dayer MJ, Jones S, Prendergast B, et al. Incidence of infective endocarditis in England, 2000-13: A secular trend, interrupted time-series analysis. Lancet 2015;385:1219-28.

3. Jankelow D, Cupido B, Zühlke L, et al. Prevention of infective endocarditis associated with dental interventions: 
South African Heart association position statement, endorsed by the South African Dental Association. SA Heart 2017;14:170-4.

4. Cahill TJ, Prendergast BD. Infective endocarditis. Lancet 2016;387:882-93.

5. Koegelenberg CFN, Doubell AF, Orth H, et al. Infective endocarditis in the Western Cape Province of South Africa: a three-year prospective study. QJM 2003;96:217-25.

6. Koshy J, Engel M, Human P, et al. Long term outcome and EuroSCORE II validation in native valve surgery for active infective endocarditis in a South African cohort. SA Heart 2018;15:116-26.

7. Reményi B, Wilson N, Steer A, et al. World Heart Federation criteria for echocardiographic diagnosis of rheumatic heart disease-an evidence-based guideline. Nat Rev Cardiol 2012;9:297-309.

8. Van Deventer J, Doubell A, Herbst P, et al. Evaluation of the SUNHEART Cardiology Outreach Programme. SA Heart 2015;12:82-6.

9. Cahill TJ, Prendergast BD. Current controversies in infective endocarditis. F1000Research 2015;4:1287.

10. Meel R, Essop MR. Striking increase in the incidence of infective endocarditis associated with recreational drug abuse in urban South Africa. S Afr Med J 2018;108:585.

11. Habib G, Lancellotti P, Antunes MJ, et al 2015 ESC Guidelines for the management of infective endocarditis. Eur Heart J 2015;36:3075-128.

12. Vogkou CT, Vlachogiannis NI, Palaiodimos L, et al. The causative agents in infective endocarditis: a systematic review comprising 33,214 cases. Eur J Clin Microbiol Infect Dis 2016;35:1227-45.

13. Moodley K, Govind CN, Peer AKC, et al. Native valve endocarditis due to Candida parapsilosis in an adult patient. South Afr J HIV Med 2013;14:138-40.

14. Arnold CJ, Johnson M, Bayer AS, et al. Candida Infective Endocarditis: an Observational Cohort Study with a Focus on Therapy. Antimicrob Agents Chemother 2015;59:2365-73.

15. Stout KK, Verrier ED. Acute Valvular Regurgitation. Circulation 2009;119:3232-41.

16. Wang A, Gaca JG, Chu VH. Management considerations in infective endocarditis: A review. JAMA 2018;320:72-83.

17. Fournier PE, Thuny F, Richet H, et al. Comprehensive Diagnostic Strategy for Blood Culture-Negative Endocarditis: A Prospective Study of 819 New Cases. Clin Infect Dis 2010;51:131-40.

18. Naidoo P, Theron G, Rangaka MX, et al. The South African Tuberculosis Care Cascade: Estimated Losses and Methodological Challenges. J Infect Dis 2017;216:S702-13.
19. Liu A, Nicol E, Hu Y, et al. Tuberculous Endocarditis. Int J Cardiol 2013;167:640-5.

20. Falk V, Baumgartner H, Bax JJ, et al. 2017 ESC/EACTS Guidelines for the management of valvular heart disease. Eur J Cardiothorac Surg 2017;52:616-64.

21. Gomes A, Glaudemans AWJM, Touw DJ, et al. Diagnostic value of imaging in infective endocarditis: a systematic review. Lancet Infect Dis 2017;17:e1-14.

22. Bedeir K, Reardon M, Ramlawi B. Infective endocarditis: Perioperative management and surgical principles. J Thorac Cardiovasc Surg 2014;147:1133-41.

23. Iversen K, Ihlemann N, Gill SU, et al. Partial Oral versus Intravenous Antibiotic Treatment of Endocarditis. N Engl J Med 2019;380:415-24.

24. Hodges KE, Hussain ST, Stewart WJ, et al. Surgical management of infective endocarditis complicated by ischemic stroke. J Card Surg 2017;32:9-13.

25. Dickerman SA, Abrutyn E, Barsic B, et al. The relationship between the initiation of antimicrobial therapy and the incidence of stroke in infective endocarditis: An analysis from the ICE Prospective Cohort Study (ICE-PCS). Am Heart J 2007;154:1086-94.

26. Angstwurm K, Borges AC, Halle E, et al. Timing the valve replacement in infective endocarditis involving the brain. J Neurol 2004;251:1220-6.

27. Rudasill SE, Sanaiha Y, Mardock AL, et al. Clinical Outcomes of Infective Endocarditis in Injection Drug Users. J Am Coll Cardiol 2019;73:559-70.

28. Naili MA, Herbst PG, Doubell AF, et al. A retrospective audit of mitral valve repair surgery at Tygerberg Hospital. SA Heart 2018;15:182-9.

29. Janson JT, Coetzee A, Rossouw G, et al. Replacing the Anterior Mitral Valve Leaflet With Autologous Jugular Vein in a Sheep Model. Ann Thorac Surg 2017;104:584-92.

30. Janson JT, Pecoraro A. Reinventing the Saphenous Vein: Reconstructing the Anterior Mitral Leaflet With a Saphenous Vein. Ann Thorac Surg 2019;107:e287-9.

31. Iung B, Rousseau-Paziaud J, Cormier B, et al. Contemporary Results of Mitral Valve Repair for Infective Endocarditis. J Am Coll Cardiol 2004;43:386-92.

32. Cahill TJ, Harrison JL, Jewell P, et al. Antibiotic prophylaxis for infective endocarditis: A systematic review and meta-analysis. Heart 2017;103:937-44.

Cite this article as: Pecoraro AJ, Doubell AF. Infective endocarditis in South Africa. Cardiovasc Diagn Ther 2020;10(2):252-261. doi: 10.21037/cdt.2019.06.03 